\title{
Clinical relevance of pulmonary non- tuberculous mycobacterial isolates in three reference centres in Belgium: a multicentre retrospective analysis
}

Yannick Vande Weygaerde ${ }^{1 *}\left(\mathbb{D}\right.$, Nina Cardinaels ${ }^{2 \dagger}$, Peter Bomans ${ }^{3}$, Taeyang Chin ${ }^{1}$, Jerina Boelens ${ }^{4}$, Emmanuel André, Eva Van Braeckel ${ }^{1 \dagger}$ and Natalie Lorent ${ }^{2 \dagger}$

\begin{abstract}
Background/objectives: Assessing the clinical relevance of non-tuberculous mycobacteria (NTM) isolated from respiratory samples can be challenging. The epidemiology and pathogenicity of NTM species vary geographically. We aimed to outline the clinical relevance and associated radiological patterns of NTM species isolated in Belgium.

Methods: We performed a retrospective multicentre analysis of all patients identified from the laboratory database with $>1$ respiratory sample growing NTM from January 2010 through December 2017. We collected clinical, radiological and microbiological data through medical record review and assessed clinical relevance according to ATS/IDSA criteria for NTM pulmonary disease (NTM-PD).

Results: Of the 384 unique patients, $60 \%$ were male, 56\% had a smoking history and $61 \%$ had pre-existing lung disease. Mycobacterium avium complex (MAC), M. gordonae and M. xenopi were the most frequently isolated species: 53,15 and $8 \%$ respectively. $43 \%$ of patients met ATS/IDSA criteria, of whom $28 \%$ presented with fibrocavitary disease. Weight loss, fever, nodular bronchiectatic and fibrocavitary lesions on chest CT, and a positive acid-fast bacilli (AFB) stain were significantly associated with NTM-PD. The species with the highest pathogenic potential were M. abscessus (11/12), M. malmoense (6/7) and M. intracellulare (41/64).
\end{abstract}

Conclusion: In our study, MAC was the most commonly isolated NTM species, but M. abscessus and M. malmoense showed the highest probability of being clinically relevant. Clinical relevance varied not only by species but also by radiological findings on chest $C T$ and AFB staining. Clinicians should consider these elements in their treatment decision making. Prospective data including clinical outcome are needed to provide more robust evidence.

Keywords: Non-tuberculous mycobacteria (NTM), Non-tuberculous mycobacterial pulmonary disease (NTM-PD), Epidemiology, Radiology

\section{Background}

Non-tuberculous mycobacteria (NTM) are ubiquitous in the natural environment and household water systems $[1,2]$. Over 180 different species have been identified, but only 32 have been reported causing human or animal disease [3].

\footnotetext{
* Correspondence: yannick.vandeweygaerde@ugent.be

${ }^{+}$Yannick Vande Weygaerde and Nina Cardinaels contributed equally as authors of this manuscript. Eva Van Braeckel and Natalie Lorent contributed equally as promotors of this manuscript.

'Department of Respiratory Medicine, Ghent University Hospital, Corneel Heymanslaan 10, B9000 Ghent, Belgium

Full list of author information is available at the end of the article
}

Lung infections (NTM related pulmonary disease or NTMPD) account for $90 \%$ of NTM related disease. Patients with pre-existing lung disease like chronic obstructive pulmonary disease (COPD), cystic fibrosis (CF), and non-CF bronchiectasis are predisposed [4-8]. To a lesser extent NTM can cause infections of skin, sinuses, lymph nodes, or even lead to disseminated disease in case of innate or acquired immunodeficiency $[5,9]$.

Worldwide pulmonary isolation of NTM is increasing and, according to some data, so is the incidence of NTM-

(c) The Author(s). 2019 Open Access This article is distributed under the terms of the Creative Commons Attribution 4.0 International License (http://creativecommons.org/licenses/by/4.0/), which permits unrestricted use, distribution, and reproduction in any medium, provided you give appropriate credit to the original author(s) and the source, provide a link to the Creative Commons license, and indicate if changes were made. The Creative Commons Public Domain Dedication waiver (http://creativecommons.org/publicdomain/zero/1.0/) applies to the data made available in this article, unless otherwise stated. 
PD [10-14]. Since NTM-PD is not a reportable disease in most countries, reliable epidemiological data are scarce.

NTM species vary between countries and regions and can even change over time, as does their respective pathogenicity [10, 12, 15-17]. Overall, species from the Mycobacterium avium complex (MAC) are the most commonly isolated NTM $[12,15,18]$. M. avium predominates in North America [12, 13], whereas in Australia $M$. intracellulare is more prevalent [19]. In the Republic of Korea $M$. intracellulare is the most prevalent species but $M$. abscessus is also quite common [20, 21]. In Europe, M. avium and M. intracellulare are almost equally prevalent, but species other than MAC are also frequently isolated which can be clinically relevant as well (M. kansasii, M. xenopi, M. malmoense, ...) $[15,18]$. There are marked regional differences in isolation frequency and pathogenic potential of different species[12, 15, 18]. Epidemiological data from Belgium are limited to isolation frequency without clinical information [17].

Assessing the clinical relevance of respiratory NTM isolates can be challenging. Intermittent isolation is frequent and spontaneous culture conversion is possible without harm to the patient. The American Thoracic Society (ATS) and the Infectious Diseases Society of America (IDSA) developed diagnostic criteria for NTMPD to guide treatment decisions, since objective markers of disease predicting which patients will develop active NTM-PD are lacking [4]. These criteria are widely implemented although based on expert opinion and barely validated [5]. Whether they can be universally applied in all patient populations remains debatable.

Besides ATS/IDSA criteria, taking into account the local epidemiology and individual species pathogenicity can facilitate treatment decision-making. This should be a well-considered process given the prolonged, potentially toxic and costly multidrug regimens used in NTMPD with relatively poor efficacy $[4,5,7]$.

We aimed to outline the epidemiology of respiratory NTM isolates in our region, assess their clinical relevance according to the ATS/IDSA criteria and explore the possibility of an association between clinical relevance and the radiological pattern on chest CT.

\section{Methods \\ Design}

We performed a multicentre, retrospective cohort study including all patients with at least one respiratory sample yielding NTM between January 2010 and December 2017 from three Belgian reference centres: University Hospitals Leuven, Ghent University Hospital and Stuivenberg Hospital Antwerp. The ethics committees of all participating centres granted approval. Informed consent was waived given the retrospective nature of the study.

\section{Study cohort}

Starting from the microbiology database, we searched for samples positive for NTM of patients aged $>18$ years from January 2010 through December 2017. We only included patients with respiratory samples defined as sputum, bronchial wash, mediastinal lymph node biopsy and lung biopsy. People living with human immunodeficiency virus (HIV) were excluded because of their different disease presentation.

\section{Data collection}

At least one investigator per centre reviewed the electronic patient record using a standardised clinical report form. We collected the following data: age, gender, race, comorbidities, smoking history, clinical presentation, radiological features at diagnosis, microbiology (NTM species, acid-fast bacilli (AFB) staining, co-isolation of Aspergillus fumigatus species complex and/or Pseudomonas aeruginosa). Clinical relevance was defined in accordance with ATS/IDSA criteria for NTM-PD i.e. presence of symptoms and radiologic abnormalities compatible with NTM lung disease and microbiological confirmation as specified in Table 1. Cases who did not meet the ATS/IDSA diagnostic criteria were only considered as respiratory isolation of an NTM without NTM-PD. For challenging cases a consensus was sought through an expert panel of 3 senior investigators. Immunodeficiency was considered a comorbidity when the patient suffered from a primary immunodeficiency or haematological malignancy, when he was treated with immunosuppressive drugs (systemic corticosteroids, tumor necrosis alpha inhibitors, cytostatic agents) or had a solid organ transplant or bone marrow transplant [9]. Radiological features were defined as 'fibrocavitary' when fibrotic and/or cavitary lesions were present and 'nodular bronchiectatic' when multiple small nodules $<5 \mathrm{~mm}$ and/or bronchiectasis were found. All other anomalies were grouped as 'other'. Respiratory samples were incubated on liquid and/or solid mycobacterial culture media. After ruling out the presence of $M$. tuberculosis complex (MTBc) using a targeted PCR

Table 1 Clinical and microbiological criteria for diagnosis of NTM-PD as proposed in the 2007 ATS/IDSA statement

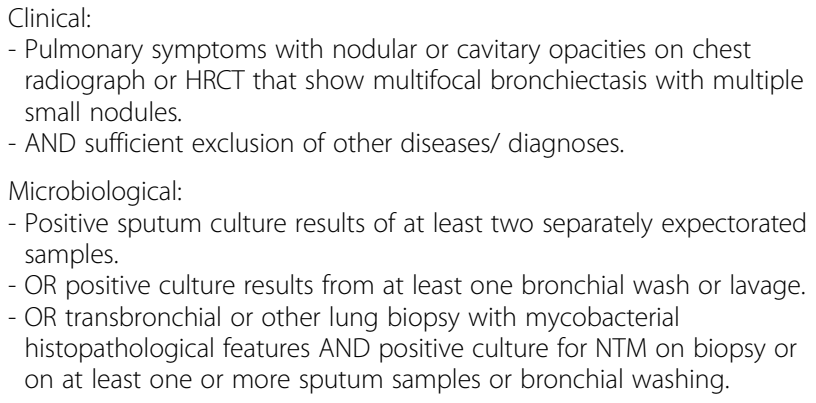

Adapted from Griffith DE, Aksamit T, Brown-Elliott BA, Catanzaro A, Daley C, Gordin F, et al. An official ATS/IDSA statement: Diagnosis, treatment, and prevention of nontuberculous mycobacterial diseases. Am J Respir Crit Care Med. 2007;175(4):367-416 
amplifying the MTBc-specific IS6110, species identification was performed at the national reference laboratory for mycobacteriology (Sciensano, Brussels, Belgium) from positive cultures and was based on a combination of techniques targeting the $16 \mathrm{~S}$ rRNA gene. PCR-based techniques included Inno-Lipa Mycobacteria v2 (Innogenetics, Gent, Belgium) and the Genotype Mycobacterium CM (Hain Lifescience $\mathrm{GmbH}$, Nehren, Germany) assays. When these tests did not provide a species identification, sequencing of the $16 \mathrm{~S}$ rRNA gene was performed as previously described [22]. Unfortunately, species and sub-species differentiation was not always achieved within the MAC and M. chelonaeabscessus complex groups.

\section{Statistics}

Continuous variables were described as medians and interquartile ranges (IQR) and categorical variables as absolute numbers and percentages of total. We compared cases with NTM-PD to cases not fulfilling the ATS/IDSA criteria using the Mann-Whitney $U$ test for not-normally distributed continuous variables (age) and $\chi^{2}$ or Fisher's exact test as appropriate (using odds ratios (OR) and 95\% confidence intervals
(CI 95\%)) for dichotomous categorical variables. A two-sided error level of $p<0.05$ was considered statistically significant. Multivariable logistic regression modelling was done including all factors associated with the outcome in univariate analysis. Starting from the full model including all co-variates, a backward stepwise selection process was performed retaining in the final model only variables with a $p$-value $<0.05$. We checked for interactions with identified risk factors for those co-variates that seemed most plausible (gender and history of previous NTM) first and conducted a stratified MantelHaenszel analysis and tested for homogeneity of the ORs across the strata. For those factors with statistically significant ( $p$-value $<0.05$ ) heterogeneity (effect modification) an interaction term was included in the logistic regression model. However, none were retained (as statistically non-significant) in the final analysis. Statistical analysis was computed with SPSS version 25.0 (SPSS, Inc., Chicago, IL, USA) and Stata version 15 (StataCorp, Texas, USA).

\section{Results}

Of the 508 unique patients in whom NTM were isolated, we identified 384 patients with at least one NTM positive

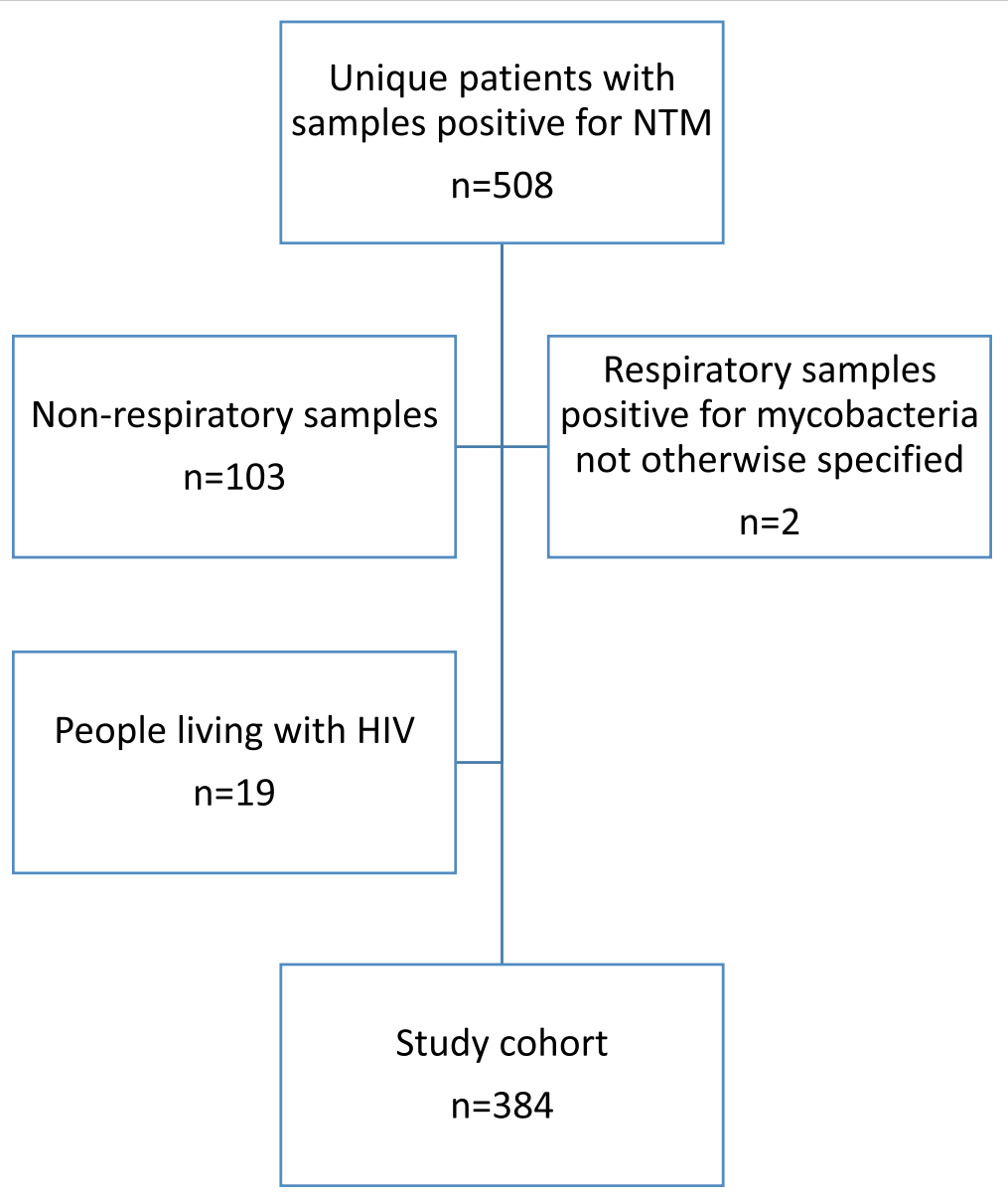

Fig. 1 Selection of our study cohort 


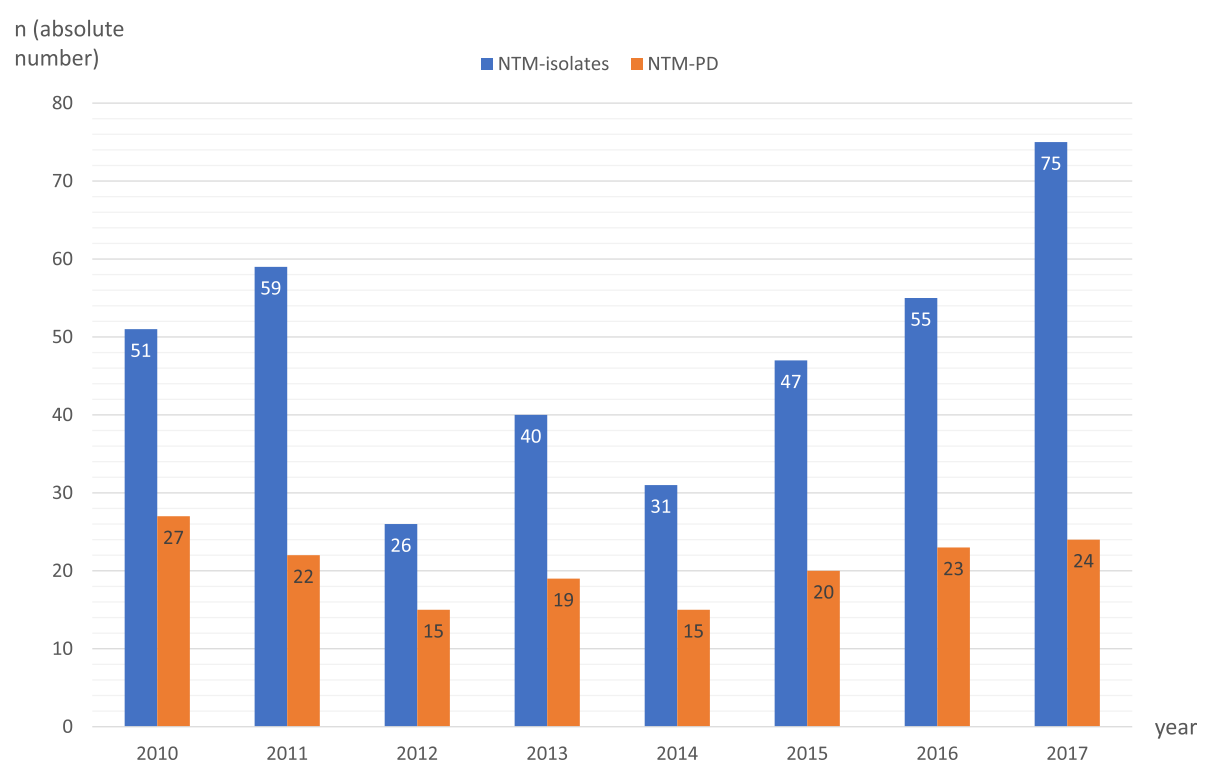

Fig. 2 New isolates and diagnoses per year. Histogram with annual detection of new patients with an NTM-yielding respiratory sample (blue) and the annual incidence of new cases of NTM-PD (orange)

respiratory sample (Fig. 1). Figure 2 shows the evolution over time of NTM respiratory isolates and NTM-PD cases. We noted an increase in the number of respiratory NTM isolates from 51 in 2010 to 75 new isolations (in unique patients) in 2017, whereas the number of new cases of NTM-PD remained approximately stable during our study period. Over $90 \%$ of the patients in our cohort were seen by a pulmonologist. Forty-three percent (165/384) of the patients fulfilled ATS/IDSA criteria for NTM-PD. Table 2 presents baseline demographic, clinical, radiological and microbiological characteristics of the total cohort as well as by fulfilment of ATS/IDSA criteria. Over half of patients were current or ex-smokers (56.5\%), 60.4\% were male, and the majority were of Caucasian descent (95.8\%), with a median (IQR) age of 65 (54-74) years. Pre-existing lung disease was present in 61\%, most commonly COPD/emphysema (41.4\%) and non-CF bronchiectasis (15.6\%). Over 20\% was immunocompromised to some extent. Besides previous episodes of NTM-PD and non-CF bronchiectasis, no other comorbidities were found to be significantly associated with NTM-PD after univariate analysis. These co-variates were not significant in multivariate analysis.

Cough (72.4\%), sputum expectoration (50\%) and dyspnoea $(48.4 \%)$ were the most frequently reported presenting symptoms. Fever (17.7\%), night sweats (7.8\%), fatigue (28.4\%), weight loss (28.1\%), and hemoptysis (16.4\%) were less common. In univariate analysis, fever (24.2\%), night sweats $(12.1 \%)$, fatigue $(38.2 \%)$, cough $(81.8 \%)$, weight loss (43\%) and haemoptysis (21.2\%) were more commonly associated with NTM-PD cases than with cases not fulfilling ATS/IDSA criteria (Table 2).
Fibrocavitary lesions were present in 63 (16.4\%) patients, whereas 188 (49\%) patients had nodular bronchiectatic changes; 16 (4.2\%) had a normal chest CT. Fibrocavitary lesions were associated with NTM-PD (OR 4.45 [2.44$8.12]$ ) as were nodular bronchiectatic lesions (OR 2.50 [1.64-3.81]).

Twenty percent of patients had positive AFB staining, which correlated significantly with ATS/IDSA criteria (OR 9.28 [4.96-17.34]). In univariate analysis (but not in multivariate analysis) co-isolation of $A$. fumigatus seemed significantly associated with NTM-PD cases (OR 2.32 [1.37-3.93]). This was not the case for co-isolation of $P$. aeruginosa (OR 1.07 [0.60-1.90]) (Table 2).

Multivariate analysis revealed that only symptoms like fever (OR 2.26 [1.12-4.56]) and weight loss (OR 2.47 [1.42-4.31]); fibrocavitary (OR 11.0 [4.74-25.57]) and nodular bronchiectatic findings (OR 7.34 [3.87-13.91]) on chest CT as well as positive AFB-staining (OR 5.20 [2.6010.42]) were independently associated with NTM-PD.

MAC was the commonest in terms of isolates in general and clinically relevant isolates (53.4\%). On individual species level, M. avium (25\%) was the most frequently isolated species followed by $M$. intracellulare $(16.7 \%)$, M. gordonae (14.6\%) and M. xenopi (7.6\%). Among the clinically relevant isolates (NTM-PD), again MAC was predominant (67.3\%); M. avium (31.5\%), M. intracellulare (24.8\%), M. xenopi (8.5\%) and M. abscessus (6.7\%) being the most frequently encountered pathogens in NTM-PD subjects (Fig. 3).

Figure 4 illustrates the pathogenic potential of the individual species according to ATS/IDSA criteria. $M$. 
Table 2 Clinical characteristics of the 384 subjects of our study cohort by ATS/IDSA criteria

\begin{tabular}{|c|c|c|c|c|c|}
\hline & Total cohort $(n=384)$ & ATS/IDSA $+(n=165)$ & ATS/IDSA - $(n=219)$ & $\mathrm{OR}^{\mathrm{a}}[\mathrm{Cl}$ 95\%] & $\mathrm{aOR}^{\mathrm{b}}[\mathrm{Cl}$ 95\%] \\
\hline Demography & n (\%) & n (\%) & n (\%) & & \\
\hline Median age in years $(\mathrm{IQR})^{\mathrm{c}}$ & $65(54-74)$ & $64(52-74)$ & $67(55-75)$ & - & - \\
\hline Male & $232(60.4 \%)$ & $94(57 \%)$ & $138(63 \%)$ & $0.78[0.51-1.17]$ & - \\
\hline Smoking history ${ }^{d}$ & $217(56.5 \%)$ & $96(58.2 \%)$ & $121(55.3 \%)$ & $1.03[0.68-1.57]$ & - \\
\hline \multicolumn{6}{|l|}{ Comorbidity $^{\mathrm{e}}$} \\
\hline Non-CF bronchiectasis & $60(15.6 \%)$ & $33(20 \%)$ & $27(12.3 \%)$ & $1.74[1.00-3.03]$ & - \\
\hline Cystic fibrosis & $24(6.3 \%)$ & $10(6.1 \%)$ & $14(6.4 \%)$ & $0.93[0.40-2.14]$ & - \\
\hline COPD/emphysema & 159 (41.4\%) & $69(41.8 \%)$ & $90(41.1 \%)$ & $1.00[0.66-1.51]$ & - \\
\hline Previous NTM disease & $31(8.1 \%)$ & $23(13.9 \%)$ & $8(3.7 \%)$ & $4.19[1.82-9.63]$ & - \\
\hline Previous TB disease & $32(8.3 \%)$ & $12(7.3 \%)$ & $20(9.1 \%)$ & $0.76[0.36-1.61]$ & - \\
\hline Pulmonary aspergillosis & $16(4.2 \%)$ & $7(4.2 \%)$ & $9(4.1 \%)$ & $1.01[0.37-2.78]$ & - \\
\hline Gastroesophageal reflux & $28(7.3 \%)$ & $8(4.8 \%)$ & $20(9.1 \%)$ & $0.50[0.21-1.16]$ & - \\
\hline Active malignancy & $63(16.4 \%)$ & $28(17 \%)$ & $35(16 \%)$ & $1.05[0.61-1.81]$ & - \\
\hline Immunodeficiency & $91(23,7 \%)$ & $44(26,7 \%)$ & $47(21.5 \%)$ & $1.30[0.81-2.09]$ & - \\
\hline Transplantation & $22(5.7 \%)$ & $9(5.5 \%)$ & $13(5.9 \%)$ & $0.90[0.37-2.15]$ & - \\
\hline Other & $127(33.1 \%)$ & $52(31.5 \%)$ & $75(34.2 \%)$ & $0.86[0.56-1.33]$ & - \\
\hline \multicolumn{6}{|l|}{ Symptomatology ${ }^{f}$} \\
\hline Fever & $68(17.7 \%)$ & $40(24.2 \%)$ & $28(12.8 \%)$ & $2.11[1.24-3.61]$ & $2.26[1.12-4.56]$ \\
\hline Night sweats & $30(7.8 \%)$ & $20(12.1 \%)$ & $10(4.6 \%)$ & $2.80[1.27-6.16]$ & - \\
\hline Fatigue/malaise & 109 (28.4\%) & $63(38.2 \%)$ & $46(21 \%)$ & $2.24[1.43-3.53]$ & - \\
\hline Sputum & $192(50 \%)$ & $90(54.5 \%)$ & $102(46.6 \%)$ & 1.31 [0.87-1.96] & - \\
\hline Chest pain & $41(10.7 \%)$ & $20(12.1 \%)$ & $21(9.6 \%)$ & $1.26[0.66-2.41]$ & - \\
\hline Cough & $278(72.4 \%)$ & 135 (81.8\%) & $143(65.3 \%)$ & $2.20[1.35-3.59]$ & - \\
\hline Dyspoea & $186(48.4 \%)$ & $77(46.7 \%)$ & 109 (49.8\%) & $0.84[0.56-1.25]$ & - \\
\hline Weight loss & $108(28.1 \%)$ & 71 (43\%) & $37(16.9 \%)$ & $3.59[2.25-5.74]$ & $2.47[1.42-4.31]$ \\
\hline Hemoptysis & $63(16.4 \%)$ & $35(21.2 \%)$ & $28(12.8 \%)$ & $1.78[1.03-3.07]$ & - \\
\hline Other & $31(8.1 \%)$ & $11(6.7 \%)$ & $20(9.1 \%)$ & $0.69[0.32-1.48]$ & - \\
\hline \multicolumn{6}{|l|}{ Radiology (chest CT) ${ }^{g}$} \\
\hline Fibrocavitary lesions & $63(16.4 \%)$ & 46 (27.9\%) & $17(7.8 \%)$ & $4.45[2.44-8.12]$ & $11.0[4.74-25.57]$ \\
\hline Nodular bronchiectatic lesions & $188(49 \%)$ & $103(54.8 \%)$ & $85(38.8 \%)$ & $2.50[1.64-3.81]$ & $7.34[3.87-13.91]$ \\
\hline Other & $124(32.3 \%)$ & $15(12.1 \%)$ & $109(49.8 \%)$ & $0.094[0.052-0.17]$ & - \\
\hline \multicolumn{6}{|l|}{ Microbiology } \\
\hline AFB staining positive & $78(20.3 \%)$ & $64(38.8 \%)$ & $14(6.4 \%)$ & $9.28[4.96-17.34]$ & $5.20[2.60-10.42]$ \\
\hline Pseudomonas aeruginosa ${ }^{h}$ & $54(14.1 \%)$ & $24(14.5 \%)$ & $30(13.7 \%)$ & $1.07[0.60-1.90]$ & - \\
\hline Aspergillus fumigatus $^{h}$ & $70(18.2 \%)$ & $42(25.5 \%)$ & $28(12.8 \%)$ & $2.32[1.37-3.93]$ & - \\
\hline
\end{tabular}

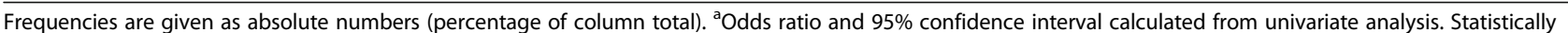
significant differences in univariate analysis (two-sided $p<0.05$ ) marked in bold. ${ }^{b}$ Adjusted odds ratio based on results from multivariate analysis. ${ }^{\mathrm{C}}$ Age as median age (IQR) in years, no statistically significant difference with Mann-Whitney $U$ test. Missing information for: ${ }^{d} 12,{ }^{f} 6$, ${ }^{g} 9$ patients respectively. ${ }^{h}$ Found as co-isolates with NTM

szulgai $(2 / 2)$ was rare but very pathogenic, followed by the more common $M$. abscessus (11/12), $M$. malmoense (6/7), M. intracellulare (41/64), M. kansasii (7/11), M. avium (52/96) and M. xenopi (14/29). M. gordonae (1/ 56), $M$. chelonae (1/9) and M. fortuitum (2/15) were rarely considered clinically relevant.
Most NTM-PD cases caused by M. kansasii (57.1\%), M. malmoense (83.3\%) and M. xenopi (71.4\%) presented with fibrocavitary disease. In contrast, the majority of cases of M. abscessus related NTM-PD presented with nodular bronchiectatic disease (81.8\%). This also applies to the majority of cases involving MAC; however, cases 


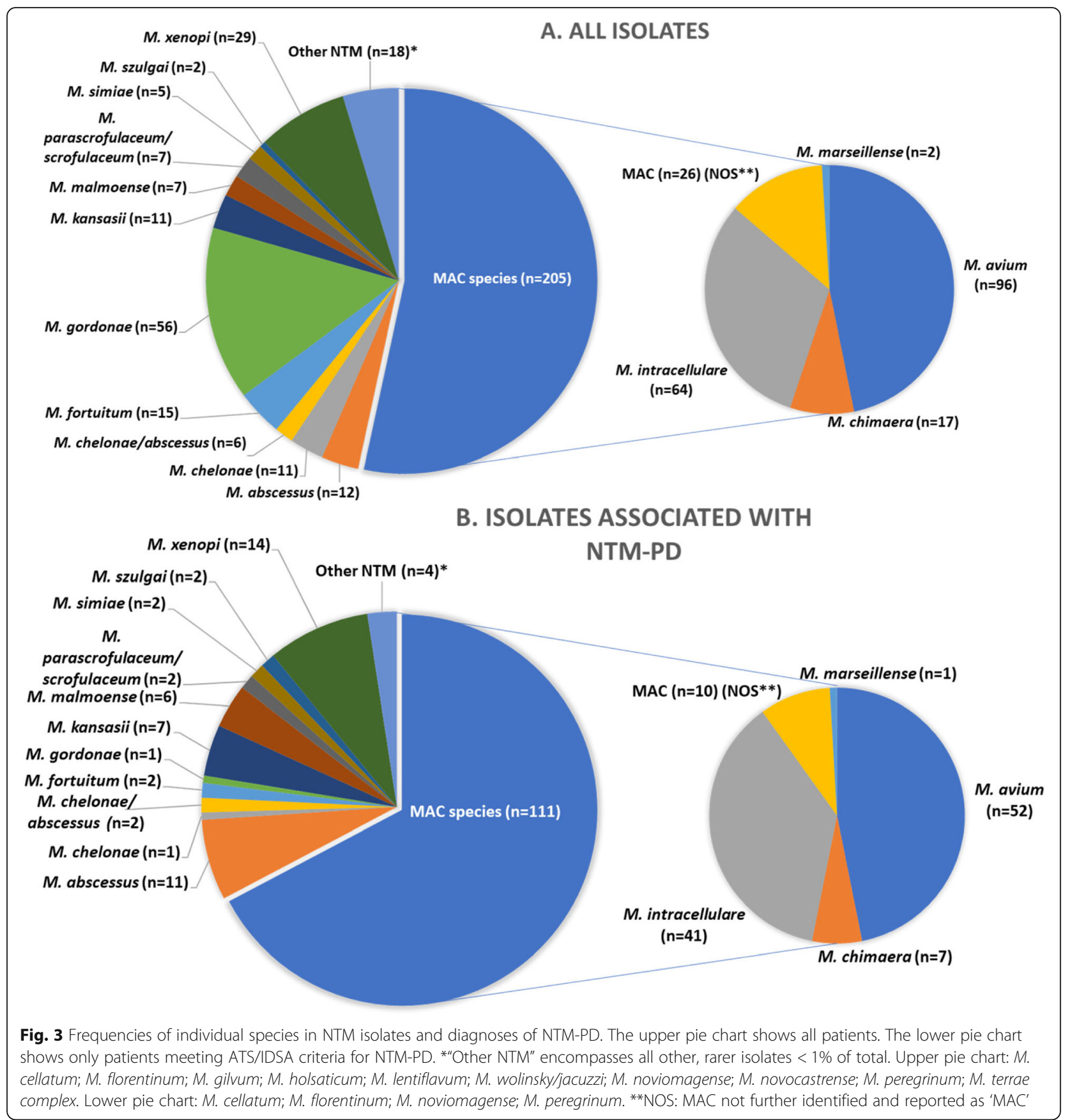

with MAC presenting with fibrocavitary lesions had a higher relative frequency of NTM-PD. When presenting with fibrocavitary changes on chest $\mathrm{CT}$ most isolated NTM were clinically significant $(46 / 63 ; 73 \%)$. As for nodular bronchiectatic lesions, this was less pronounced $(103 / 188,55 \%)$. When neither fibrocavitary nor nodular bronchiectatic lesions were present on chest CT, the isolated NTM was most often not considered clinically relevant $(15 / 124 ; 12 \%)$ with few exceptions as presented in Fig. 5. As an example, M. xenopi was clinically relevant in 14 of $29(48 \%)$ isolates. When associated with fibrocavitary lesions clinical relevance increased to $10 / 14$ (71\%), whereas in the presence of nodular bronchiectatic disease this was $3 / 6$ (50\%). When neither were present, M. xenopi isolation was mostly not relevant (only $1 / 9$ ).

\section{Discussion}

To the best of our knowledge, the present study is the first to describe clinical and radiographic features in relation to species-specific pathogenic potential of respiratory NTM 


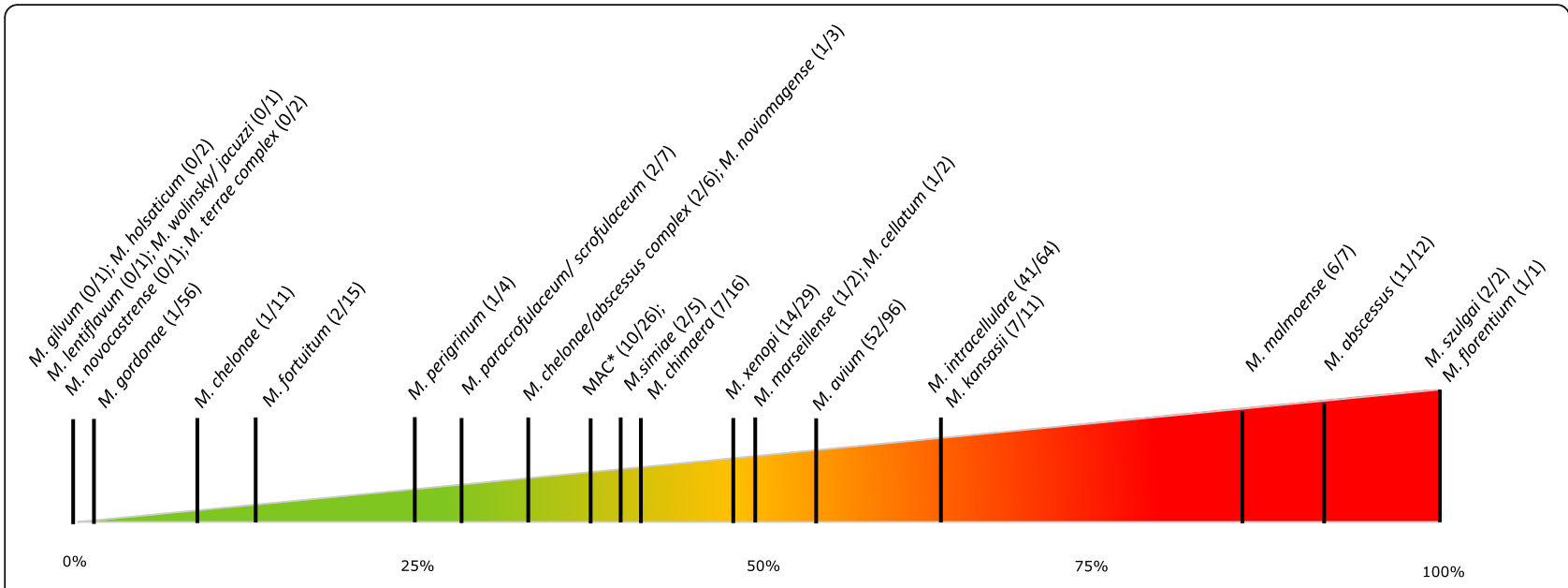

Fig. 4 Ratio of clinical significance. Clinical significance per species; presented as a percentage based on the number of cases meeting ATS/IDSA criteria for NTM-PD and the total number of isolates per species. MAC and M. chelonae/abscessus complex, not further specified, were regarded separately. On the left, species of low virulence are found and on the right the most pathogenic species. Figure concept van Ingen et al., Thorax 2009, with permission

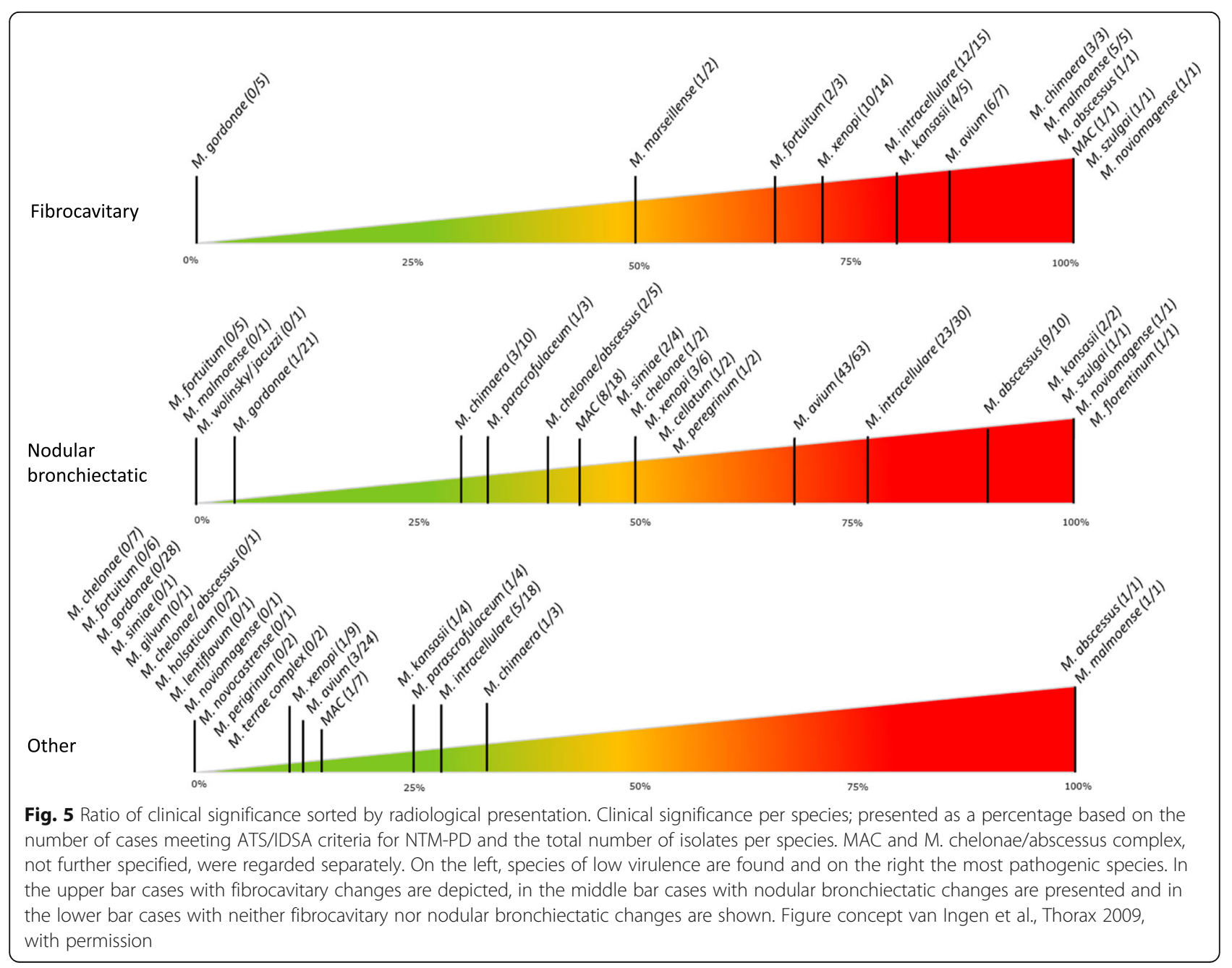


isolates in Belgium. The frequency of NTM-PD remained stable over an 8-year time period in the three participating reference centres, despite increasing isolation frequency. NTM isolates were clinically significant in $43 \%$ of the patients. Symptoms like fever and weight loss, fibrocavitary and nodular bronchiectatic lesions on chest CT and a positive AFB stain were significantly associated with NTM-PD. MAC was the commonest mycobacterial isolate and pathogen in our cohort.

Although there was an annual increase in new NTM isolates, numbers of newly diagnosed NTM-PD remained stable. This evolution over time is in line with study findings in Denmark and the Netherlands [23, 24], whereas reports from the UK, Germany, Canada and the USA report an increasing incidence of NTM-PD [11, 13, 14, 25, 26]. The increasing isolation of NTM from respiratory samples may be partly due to a rising awareness and screening, but may also be explained by environmental factors or an ageing population with more comorbidities and predisposition to NTM-PD. However, the real incidence of NTM-PD remains uncertain since NTM-PD is not a reportable disease in most countries. Therefore, longitudinal and prospective registries could be very informative concerning the true incidence of NTM-PD.

Less than half of pulmonary NTM isolates are considered clinically relevant according to the ATS/IDSA criteria. Over $40 \%$ of patients in our cohort met the ATS/ IDSA criteria, in contrast to lower reported numbers of 25-33\% in similar studies from the Netherlands, the UK and South Korea [23, 27, 28]. This might be explained by the nature of referrals and underlying disease severity.

Patients in our study cohort were predominantly male and middle-aged, which is in concordance with some previous reports $[6,23]$ but in contrast to reports from the US and Japan where a different morphotype (white, middle-aged females) is described [12, 29, 30]. Preexisting lung disease such as COPD and bronchiectasis were the most common comorbidities. Chronic respiratory disease is an important risk factor for NTM-PD, making it a pulmonologists issue: it has been associated with a 16-fold increased risk in a Danish population-based casecontrol study [31]. The finding was confirmed by Marras et al, reporting a nine times higher incidence of NTM-PD in COPD [8]. Closer surveillance for NTM in patients with pulmonary disease may be warranted.

Diagnosis of NTM-PD according to ATS-IDSA criteria can be challenging as symptoms are often nonspecific and partly overlapping with underlying illness. Fever, night sweats and weight loss tend to be present in advanced NTM-PD [4]. Similarly in our study, fever and weight loss were independently associated with NTMPD. Cough and fatigue also tended to be more frequently reported in patients with NTM-PD similar to findings from Aksamit et al., but these are rather non- specific symptoms which are often related to underlying diseases [4, 29]. The presence of nodular bronchiectatic lesions and especially fibrocavitary lesions on chest CT were significantly associated with NTM-PD. In the absence of either fibrocavitary or nodular bronchiectatic lesions, isolates were mostly not clinically relevant, a finding corroborating the ATS/IDSA criteria. Fibrocavitary lesions are a marker of severe NTM-PD with worse prognosis [4, 32, 33]. Similarly, the majority of patients in our cohort with respiratory samples positive on AFB staining suffered from NTM-PD. This is in line with previous studies, where AFB stain positivity has been associated with a higher bacterial burden, more severe NTM-PD and poor outcome [5, 33]. A. fumigatus coisolation was also associated with NTM-PD in univariate analysis as suggested in previous studies by Kunst et al. and Provoost et al. [34, 35]. The value of this finding remains unclear as in multivariate analysis this factor was no longer deemed significant. Is A. fumigatus co-isolation a risk factor or the consequence of NTM-PD? It could also be related to underlying lung disease or immunosuppression [34-36]. Prospective studies or registries could identify true risk factors or indicators which could help refining the current ATS/IDSA criteria for establishing the diagnosis of NTM-PD.

With MAC accounting for more than half of the isolates, its prevalence in our study is comparable with a 2008 report from the Belgian national reference lab in which $38 \%$ of all isolates were MAC [18]. In other European studies, MAC was the predominant species as well, although regional differences were noted $[15,18]$. M. avium, M. intracellulare, $M$. gordonae and $M$. xenopi were the most frequently isolated species, in accordance with a national report by Soetaert et al. where $M$. avium, $M$. intracellulare and $M$. xenopi accounted for 20, 21 and 15\% of all (pulmonary and other) isolates respectively [17]. Of note, the same group previously showed that $M$. chimaera was frequently misidentified as $M$. intracellulare [22]. In our report we did not systematically re-evaluate these very closely related species. The difference for $M$. xenopi could be explained as our cohort did not properly cover the coast nor the southern half of the country which have a distinct environment. $M$. xenopi is a species mainly found in Europe with high concentrations in Southern Europe and along the English Channel [18, 37]. Accounting for only 3\% of isolates, $M$. abscessus was rather rare which is similar to other European reports $[12,15]$. Prevalence of $M$. abscessus isolates is substantially higher in Asia (16\%) and Oceania (12\%). These differences can be explained by different environmental factors and/or changing mycobacterial fauna over time $[12,15,17,18]$.

MAC was the main culprit (67.3\%) for NTM-PD in this study cohort (Fig. 3). The position of MAC as the main cause of NTM-PD is established worldwide although 
frequencies vary largely between studies $[12,15]$. Of individual species $M$. szulgai, $M$. abscessus, $M$. malmoense, $M$. kansasii, M. intracellulare and M. avium were clinically relevant in more than $50 \%$ of cases, suggesting a higher pathogenic potential than other species (Fig. 4). Compared to a Dutch study by Van Ingen et al., there are several similarities but also some differences [23]. Our M. abscessus and $M$. intracellulare isolates were more frequently considered clinically relevant than their Dutch counterparts (91.6\% versus 33 and $64 \%$ versus $12.5 \%)$. In our cohort $M$. intracellulare had a higher probability of clinical relevance than $M$. avium (64 and $54.2 \%$ respectively) in contrast to the Dutch report (12.5 and 40.7\%) [23]. Our findings, however, are in line with other reports about a higher pathogenicity of $M$. intracellulare [15, 38]. These differences support the hypothesis of regional variation and illustrate the value of local data.

Clinical relevance varies not only by species but also by radiological presentation. Clinically significant isolates of M. kansasii, M. malmoense and M. xenopi mostly presented with fibrocavitary changes while $M$. abscessus and MAC related NTM-PD more often presented with nodular bronchiectatic changes, which has already been described [39-41]. Our findings corroborate previous suggestions that when assessing NTM clinical relevance, integration of radiological findings and species identification is useful [39, 40].

Our study has several limitations inherent to the retrospective study design. Selection bias cannot be excluded since data were collected from three major reference centres receiving the more complex cases. Additionally, increased awareness over time and more intense surveillance protocols for patients with pulmonary disease may have led to overestimation of NTM-PD risk. Our data are limited in scope, hence caution is warranted in generalising the findings. The lack of outcome data limit the predictive value of clinical significance.

\section{Conclusion}

NTM are widely distributed environmental opportunistic pathogens which can cause significant morbidity and mortality especially in patient with underlying pulmonary conditions. In our cohort MAC species were the most frequently isolated, but $M$. abscessus and M. malmoense had a higher ratio of being clinically relevant when isolated. Because of geographical differences in isolates and clinical relevance between species, it is important to acknowledge local epidemiology as these data can be complimentary to the ATS/IDSA criteria in assisting clinicians in their treatment decision. Based on our study results, we suggest an added value for the AFB staining results, species identification and chest CT findings in the clinical decision-making process. Large multicentre prospective studies with standardized microbiological detection methods and clinical assessment reporting tools are highly needed.

\section{Abbreviations}

AFB: Acid-fast bacilli; ATS: American Thoracic Society; CF: Cystic fibrosis; Cl 95\%: 95\% confidence interval; COPD: Chronic obstructive pulmonary disease; HIV: Human immunodeficiency virus; IDSA: Infectious Diseases Society of America; IQR: Interquartile ranges; MAC: Mycobacterium avium complex; MTBc: Mycobacterium tuberculosis complex; NTM: Non-tuberculous mycobacteria; NTM-PD: Non-tuberculous mycobacterial pulmonary disease; OR: Odds ratio

\section{Acknowledgements}

We sincerely thank Jakko van Ingen (Radboud UMC Nijmegen) for reviewing and discussing our data.

\section{Authors' contributions}

YVDW (corresponding author): data gathering, analysis of data, writing of manuscript, figures, implementation of reviewers' remarks, preparation and submission of initial and revised manuscript. NC: data gathering, analysis of data, writing of manuscript. PB: ethics approval ZNA Stuivenberg, data gathering, critical review of manuscript. TC: data gathering, analysis of data, review of manuscript. EA: data gathering, critical review of manuscript. JB: data gathering, critical review of manuscript. EVB: ethics approval Ghent University Hospital, review of data analysis, multiple reviews of manuscript. NL: ethics approval central committee University Hospitals Leuven, review of data analysis and multivariate analysis, multiple reviews of manuscript. All authors read and approved the final manuscript.

\section{Funding}

No funding or grants were allocated to this study.

\section{Availability of data and materials}

The dataset used and/analysed during the current study is available from the corresponding author on reasonable request.

\section{Ethics approval and consent to participate}

University Hospitals Leuven

Central Ethics Committee: Ethics Committee Research UZ/ KU Leuven, Belgium

Approval code: $\mathbf{S 6 1 1 8 8}$

Ghent University Hospital

Ethics Committee of the Ghent University Hospital, Belgium

Approval code: EC/2018/0356

ZNA Stuivenberg

Commissie voor Medische Ethiek ZNA - Institutional Review Board ZNA/

OCMW Antwerpen, Belgium:

Approval code: 5064

Statement on the need for informed consent:

The need for informed consent was waived because of the exclusively retrospective nature of the study. Non-tuberculous mycobacteria are frequently isolated from respiratory samples as they are ubiquitous in the environment and often not related to pathology. Some people might have been unaware of insignificant NTM positive isolates and asking for consent might have caused unnecessary distress and unease. Some patients included, had already died, mostly of other causes and asking consent to the next of kin might have caused additional, unnecessary distress and sorrow. Furthermore, data were analysed anonymously.

\section{Competing interests}

The authors declare that they have no competing interests.

\section{Author details}

${ }^{1}$ Department of Respiratory Medicine, Ghent University Hospital, Corneel Heymanslaan 10, B9000 Ghent, Belgium. ²Department of Respiratory

Diseases, University Hospitals Leuven, Herestraat 49, B3000 Leuven, Belgium. ${ }^{3}$ Department of Pneumology, Antwerp Hospital Network Stuivenberg, Lange Beeldekensstraat 267, B2060 Antwerp, Belgium. ${ }^{4}$ Department of Laboratory Medicine, Department of Clinical Chemistry, Microbiology and Immunology, Ghent University Hospital, Corneel Heymanslaan 10, B9000 Ghent, Belgium. ${ }^{5}$ Laboratory of Clinical Bacteriology and Mycology, Department of

Microbiology, Immunology and Transplantation, University Hospitals Leuven, Herestraat 49, Leuven B3000, Belgium. 
Received: 23 August 2019 Accepted: 29 November 2019

Published online: 17 December 2019

\section{References}

1. Falkinham JO. Environmental sources of nontuberculous mycobacteria. Clin Chest Med. 2015:36:35-41.

2. Gebert MJ, Delgado-Baquerizo M, Oliverio AM, Webster TM, Nichols LM, Honda JR, et al. Ecological analyses of mycobacteria in showerhead biofilms and their relevance to human health. MBio. 2018;9:1-15.

3. Gupta RS, Lo B, Son J. Phylogenomics and comparative genomic studies robustly support division of the genus Mycobacterium into an emended genus Mycobacterium and four novel genera. Front Microbiol. 2018;9:67.

4. Griffith DE, Aksamit T, Brown-Elliott BA, Catanzaro A, Daley C, Gordin F, et al. An official ATS/IDSA statement: diagnosis, treatment, and prevention of nontuberculous mycobacterial diseases. Am J Respir Crit Care Med. 2007; 175:367-416.

5. Haworth CS, Banks J, Capstick T, Fisher AJ, Gorsuch T, Laurenson IF, et al. British Thoracic Society guidelines for the management of non-tuberculous mycobacterial pulmonary disease (NTM-PD). Thorax. 2017;72(Suppl 2):ii1 LP-ii64

6. Andréjak C, Thomsen V $\varnothing$, Johansen IS, Riis A, Benfield TL, Duhaut $P$, et al. Nontuberculous pulmonary mycobacteriosis in Denmark: incidence and prognostic factors. Am J Respir Crit Care Med. 2010;181:514-21.

7. Floto RA, Olivier KN, Saiman L, Daley CL, Herrmann JL, Nick JA, et al. US Cystic Fibrosis Foundation and European cystic fibrosis society consensus recommendations for the management of non-tuberculous mycobacteria in individuals with cystic fibrosis. Thorax. 2016:71:i1-22.

8. Marras TK, Campitelli MA, Kwong JC, Lu H, Brode SK, Marchand-Austin A, et al. Risk of nontuberculous mycobacterial pulmonary disease with obstructive lung disease. Eur Respir J. 2016;48:928-31.

9. Henkle E, Winthrop KL. Nontuberculous mycobacteria infections in immunosuppressed hosts. Clin Chest Med. 2015;36:91-9.

10. Adjemian J, Olivier KN, Prevots DR. Epidemiology of pulmonary Nontuberculous mycobacterial sputum positivity in patients with cystic fibrosis in the United States, 2010-2014. Ann Am Thorac Soc. 2018;15:817-26.

11. Ringshausen FC, Wagner D, de Roux A, Diel R, Hohmann D, Hickstein L, et al. Prevalence of Nontuberculous mycobacterial pulmonary disease, Germany, 2009-2014. Emerg Infect Dis. 2016;22:1102-5.

12. Prevots DR, Marras TK. Epidemiology of human pulmonary infection with Nontuberculous mycobacteria. Clin Chest Med. 2015;36:13-34.

13. Marras TK, Mendelson D, Marchand-Austin A, May K, Jamieson FB Pulmonary nontuberculous mycobacterial disease, Ontario, Canada, 19982010. Emerg Infect Dis. 2013;19:1889-91.

14. Adjemian J, Frankland TB, Daida YG, Honda JR, Olivier KN, Zelazny A, et al. Epidemiology of Nontuberculous mycobacterial lung disease and tuberculosis, Hawaii, USA. Emerg Infect Dis. 2017;23:439-47.

15. Zweijpfenning S, Ingen J, Hoefsloot W. Geographic distribution of Nontuberculous mycobacteria isolated from clinical specimens: a systematic review. Semin Respir Crit Care Med. 2018;39:336-42.

16. van Ingen J, Hoefsloot W, Dekhuijzen PNR, Boeree MJ, van Soolingen D. The changing pattern of clinical Mycobacterium avium isolation in the Netherlands. Int J Tuberc Lung Dis. 2010:14:1176-80.

17. Soetaert K, Subissi L, Ceyssens P-J, Vanfleteren B, Chantrenne M, Asikainen T, et al. Strong increase of true and false positive mycobacterial cultures sent to the National Reference Centre in Belgium, 2007 to 2016. Eurosurveillance. 2019;24:2015-9.

18. Hoefsloot W, Van Ingen J, Andrejak C, Ängeby K, Bauriaud R, Bemer P, et al. The geographic diversity of nontuberculous mycobacteria isolated from pulmonary samples: an NTM-NET collaborative study. Eur Respir J. 2013;42:1604-13.

19. Thomson RM. NTM working group at Queensland TB control Centre and Queensland mycobacterial reference laboratory. Changing epidemiology of pulmonary nontuberculous mycobacteria infections. Emerg Infect Dis. 2010;16:1576-83.

20. Koh W-J, Chang B, Jeong B-H, Jeon K, Kim S-Y, Lee NY, et al. Increasing recovery of Nontuberculous mycobacteria from respiratory specimens over a 10-year period in a tertiary referral Hospital in South Korea. Tuberc Respir Dis (Seoul). 2013;75:199-204.

21. Kim N, Yi J, Chang CL. Recovery rates of non-Tuberculous mycobacteria from clinical specimens are increasing in Korean tertiary-care hospitals. J Korean Med Sci. 2017;32:1263-7.
22. Soetaert $K$, Vluggen C, André E, Vanhoof R, Vanfleteren B, Mathys V. Frequency of Mycobacterium chimaera among Belgian patients, 2015. J Med Microbiol. 2016;65:1307-10.

23. Van Ingen J, Bendien SA, De Lange WCM, Hoefsloot W, Dekhuijzen PNR, Boeree $\mathrm{MJ}$, et al. Clinical relevance of non-tuberculous mycobacteria isolated in the Nijmegen-Arnhem region, The Netherlands. Thorax. 2009;64:502-6.

24. Hermansen TS, Ravn P, Svensson E, Lillebaek T. Nontuberculous mycobacteria in Denmark, incidence and clinical importance during the last quarter- century. Sci Rep. 2017;7:6696.

25. Shah NM, Davidson JA, Anderson LF, Lalor MK, Kim J, Thomas HL, et al. Pulmonary Mycobacterium avium-intracellulare is the main driver of the rise in non-tuberculous mycobacteria incidence in England, Wales and Northern Ireland, 2007-2012. BMC Infect Dis. 2016;16:1-6.

26. Adjemian J, Olivier KN, Seitz AE, Holland SM, Prevots DR. Prevalence of nontuberculous mycobacterial lung disease in U.S. medicare beneficiaries. Am J Respir Crit Care Med. 2012;185:881-6.

27. Schiff HF, Jones S, Achaiah A, Pereira A, Stait G, Green B. Clinical relevance of non-tuberculous mycobacteria isolated from respiratory specimens: seven year experience in a UK hospital. Sci Rep. 2019;9:1730.

28. Koh W-J, Kwon OJ, Jeon K, Kim TS, Lee KS, Park YK, et al. Clinical significance of nontuberculous mycobacteria isolated from respiratory specimens in Korea. Chest. 2006:129:341-8.

29. Aksamit TR, O'Donnell AE, Barker A, Olivier KN, Winthrop KL, Daniels MLA, et al. Adult patients with bronchiectasis. Chest. 2017;151:982-92.

30. Hayashi M, Takayanagi N, Kanauchi T, Miyahara Y, Yanagisawa T, Sugita Y. Prognostic factors of $634 \mathrm{HIV}$-negative patients with Mycobacterium avium complex lung disease. Am J Respir Crit Care Med. 2012;185:575-83.

31. Andréjak C, Nielsen $R$, Thomsen VØ, Duhaut P, Sørensen HT, Thomsen RW. Chronic respiratory disease, inhaled corticosteroids and risk of nontuberculous mycobacteriosis. Thorax. 2013;68:256-62.

32. Ito Y, Hirai T, Maekawa K, Fujita K, Imai S, Tatsumi S, et al. Predictors of 5year mortality in pulmonary Mycobacterium avium-intracellulare complex disease. Int J Tuberc Lung Dis. 2012:16:408-14.

33. Hwang JA, Kim S, Jo KW, Shim TS. Natural history of Mycobacterium avium complex lung disease in untreated patients with stable course. Eur Respir J. 2017:49(3)

34. Kunst $\mathrm{H}$, Wickremasinghe $\mathrm{M}$, Wells A, Wilson R. Nontuberculous mycobacterial disease and Aspergillus-related lung disease in bronchiectasis. Eur Respir J. 2006;28:352-7.

35. Provoost J, Valour F, Gamondes D, Roux S, Freymond N, Perrot E, et al. A retrospective study of factors associated with treatment decision for nontuberculous mycobacterial lung disease in adults without altered systemic immunity. BMC Infect Dis. 2018:18:659.

36. Geurts K, Zweijpfenning SMH, Pennings LJ, Schildkraut JA, Boeree MJ, Magis-Escurra C, et al. Nontuberculous mycobacterial pulmonary disease and Aspergillus co-infection: Bonnie and Clyde? Eur Respir J. 2019;54:1900117.

37. Martín-Casabona N, Bahrmand AR, Bennedsen J, Østergaard Thomsen V, Curcio M, Fauville-Dufaux M, et al. Non-tuberculous mycobacteria: patterns of isolation. A multi-country retrospective survey. Int J Tuberc Lung Dis. 2004:8:1186-93.

38. Boyle DP, Zembower TR, Reddy S, Qi C. Comparison of clinical features, virulence, and relapse among Mycobacterium avium complex species. Am J Respir Crit Care Med. 2015;191:1310-7.

39. Matveychuk A, Fuks L, Priess R, Hahim I, Shitrit D. Clinical and radiological features of Mycobacterium kansasii and other NTM infections. Respir Med. 2012:106:1472-7.

40. Koh W-J, Lee KS, Kwon OJ, Jeong YJ, Kwak S-H, Kim TS. Bilateral bronchiectasis and bronchiolitis at thin-section CT: diagnostic implications in nontuberculous mycobacterial pulmonary infection. Radiology. 2005:235:282-8.

41. Marras TK, Wagnetz U, Jamieson FB, Patsios DA. Chest computed tomography predicts microbiological burden and symptoms in pulmonary Mycobacterium xenopi. Respirology. 2013;18:92-101.

\section{Publisher's Note}

Springer Nature remains neutral with regard to jurisdictional claims in published maps and institutional affiliations. 